\title{
Molecular Docking and Dynamics Simulation Study of Telomerase Inhibitors as Potential Anti-Cancer Agents
}

\author{
Sherin D R, Thanathu Krishnan Manojkumar, R. Prakash Chandran, Sobha V Nair
}

Submitted date: 04/05/2020 - Posted date: 07/05/2020

Licence: CC BY-NC-ND 4.0

Citation information: D R, Sherin; Manojkumar, Thanathu Krishnan; Chandran, R. Prakash; Nair, Sobha V (2020): Molecular Docking and Dynamics Simulation Study of Telomerase Inhibitors as Potential Anti-Cancer Agents. ChemRxiv. Preprint. https://doi.org/10.26434/chemrxiv.12241166.v1

Normal cells' genomic identity is protected by telomeres and sometimes chromosomal instability was observed due to shortening of telomerase because of successive cell divisions. Reports indicate that telomerase length is crucial in determining telomerase activity which in turn leads to cancer initiation. It is reported that telomere length regulation has been identified as a plausible strategy for cancer diagnostics and treatment. In the present MS, we explored the telomerase inhibitory activity of catechin analogues and it's oligomers using computational methods. The structural properties of different ligands discussed in the MS were computed using density functional theory. Conformational effect of different chromene subunit such as $2 \mathrm{R}, 3 \mathrm{R}$ conformations were explored using computational methods. The stereochemical contributions to receptor binding such as intra ligand $\pi$-interactions of these ligands were also investigated. We herein propose that these stereochemical aspects of catechins and their oligomers as the most vital factor deciding the effective binding with the $\mathrm{N}$-terminal domain of telomerase which is an efficient strategy in cancer therapy.

File list (1) 


\title{
Molecular Docking and Dynamics Simulation Study of Telomerase Inhibitors as Potential Anti-cancer Agents
}

\author{
D. R. Sherin ${ }^{\mathrm{a}, *}$, T. K. Manojkumar, ${ }^{\mathrm{a}, *}$ R. C. Prakash ${ }^{\mathrm{b}}$ and V. N. Sobha ${ }^{\mathrm{c}}$ \\ ${ }^{\mathrm{a} C e n t r e ~ f o r ~ C o m p u t a t i o n a l ~ M o d e l i n g ~ a n d ~ D a t a ~ E n g i n e e r i n g ~}$ \\ Indian Institute of Information Technology and Management-Kerala, \\ Thiruvananthapuram, Kerala, India \\ ${ }^{b}$ M.M.N.S.S. College, Kottiyam, University of Kerala, Kerala \\ 'School of Biotechnology, Amrita Vishwa Vidyapeetham, Amritapuri, Kollam, Kerala \\ sherin.dr@iiitmk.ac.in \& manojtk@iiitmk.ac.in
}

\begin{abstract}
Normal cells' genomic identity is protected by telomeres and sometimes chromosomal instability was observed due to shortening of telomerase because of successive cell divisions. Reports indicate that telomerase length is crucial in determining telomerase activity which in turn leads to cancer initiation. It is reported that telomere length regulation has been identified as a plausible strategy for cancer diagnostics and treatment. In the present MS, we explored the telomerase inhibitory activity of catechin analogues and it's oligomers using computational methods. The structural properties of different ligands discussed in the MS were computed using density functional theory. Conformational effect of different chromene subunit such as $2 \mathrm{R}$, 3R conformations were explored using computational methods. The stereochemical contributions to receptor binding such as intra ligand $\pi-$ interactions of these ligands were also investigated. We herein propose that these stereochemical aspects of catechins and their oligomers as the most vital factor deciding the effective binding with the $\mathrm{N}$-terminal domain of telomerase which is an efficient strategy in cancer therapy.
\end{abstract}

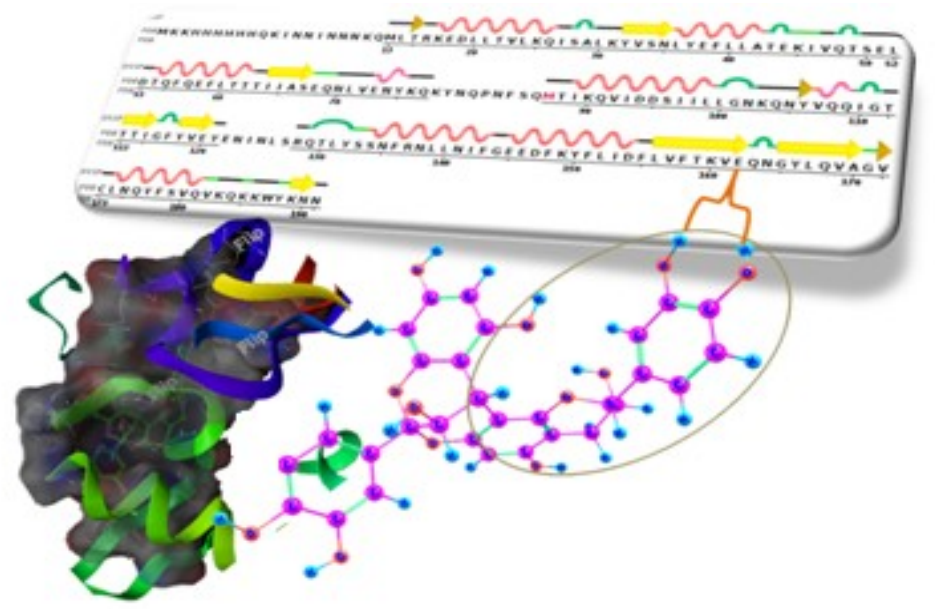

KEYWORDS: Catechins, cancer, telomerase, docking, dynamics

\section{Introduction}

Regulation of telomere length in replicative senescence by inhibiting telomerase has currently been highlighted as a potential strategy for cancer diagnostics as well as chemothrapeutics. ${ }^{1-3}$ Telomerase is a ribonucleoprotein reverse transcriptase, composed of an RNA template (TER or hTR) and a catalytic protein subunit (TERT), which in turn is formed by the alignment of four major functional domains- the TERT N-terminal domain (TEN), the TERT RNA 
binding domain (TRBD), the reverse transciptase domain (RT) and the C-terminal extension (CTE) ${ }^{4-7}$ Inhibition of telomerase is also effective in regulating proliferation of endometriosis and has been considered as a potential nonhormonal curative strategy in the pathology of endometriosis. ${ }^{8-11}$ Telomerase inhibition by amyloid beta (A $\beta$ ) binding has been reported to play a crucial role in cellular senescence and thereby fighting against Alzheimer's disease (AD). ${ }^{12}$ Thus telomerase is an important target for unwanted cellular growth, age related diseases such as $\mathrm{AD}$, endometriosis as well as in cancer therapy. ${ }^{13-16}$

The most promising methods for telomerase inhibition involve the direct disruption of capping telomeres using telomerase inhibitors along with conventional chemotherapeutics. ${ }^{17-20}$ Talari et al. reported a molecular docking approach in pharmacophore based virtual screening of new telomerase inhibitors and suggested that the TEN domain anchor site is a useful template in rational inhibitor design. ${ }^{21}$ Chen et al. demonstrated the importance of major natural products in telomerase maintenance. ${ }^{22}$ Our survey of literature revealed that role of flavanoids as telomerase inhibitors were discussed recently, but not specifically investigated. ${ }^{23-28}$ Catechins, a series of low molecular weight polyphenols that primarily consist of flavan-3-ol monomers, are present in leaves, fruits and vegetables. Takanshi et al. recently reported the anticancer activity of epicatechin oligomers longer than trimers. This will inflame us to think about the anticancer activity, especially by telomerase inhibition of catechinsuptotrimers. ${ }^{29}$ In the present work, we have explored the scope of inhibiting human telomerase TEN domain effectively by catechins. The stereochemistry and mechanistic aspects of the interaction between catechin ligands and telomerase has also been investigated. Computational simulations based on virtual screening, molecular dynamics and electronic structural methods were carried out to find out an effective output from the various conformers of catechin.

\section{Materials and methods}

\subsection{Preparation of protein and active site identification}

The crystallized TEN domain of telomerase with 2.22 $\AA$ resolution (PDB ID:2B2A) was obtained from RCSB Protein Data Bank. ${ }^{6}$ It was optimized and minimized using protein preparation wizard of Maestro, v10.1, Schrodinger suite, LLC, New York, NY, 2015. ${ }^{30}$ Sitemap, v3.4, Schrodinger, LLC, New York, NY, 2015 was used to identify active site of 2B2A receptor. The grid was generated for cleaned PDB file using the module available with Glide v6.6, Schrodinger, LLC, New York, NY, 2015. ${ }^{31}$ The dimensions of the grid box for 2B2A were kept around the centroid of active site of workspace ligand (X=61.61, $Y=61.12, \mathrm{Z}=13.61)$.

\subsection{Molecular docking}

The 3D structure of selected catechins-monomers, dimers and trimers were downloaded from PubChem open chemistry database (https://pubchem.ncbi.nlm.nih.gov). Different conformational catechins were optimized at B3LYP/6-311++G** level (Supporting Information, S5-S17) of Density functional Theory (DFT). These optimized geometries were loaded into workspace of Maestro project and all the possibleconformers were generated using LigPrep module of Schrodinger 2015 under OPLS-2005 forcefield. In silico screening and docking studies of the most stable conformers of 22 ligands on the 2B2A receptor was carried out in the active site of TEN domain of telomerase. Docking of ligands with prepared PDB file was carried out using Glideprogramme of Schrodinger suite. The interaction of the ligands with receptors were analysed on the basis of scoring functions- G-score(Glide score) and D-score (Dock score) through XP-visualizer and interaction diagrams.

\subsection{ADMET studies}

The ligands were screened by qikproptool of Schrodinger 2015 and the data were analysed for the suitablity on the basis of ADME/T (adsorption, distribution, metabolism and excretion/toxicity) and Ro5. The values are compared 
with the range obtained from QikProp manual. All the monomers and dimers with appreciable range of pharmacokinetic parameters interpreted by qikprop values are assigned for further studies.

\subsection{MM-GBSA for Binding Free Energy prediction}

The relative binding affinity of ligands $(\mathrm{kcal} / \mathrm{mol})$ were calculated by MM-GBSA, which may not in agreement with experimental values. A more negative value denotes stronger binding. It generates a lot of energy properties like that of the ligand, receptor, and complex structures as well as energy differences relating to strain and binding, and are divided into contributions from various terms in the energy expression.

Prime MMGBSA DG bind, the binding free energy, is calculated with the equation:

$$
\Delta \mathrm{G}(\text { bind })=\mathrm{E}_{\text {complex(minimized) }}-\left(\mathrm{E}_{\text {ligand(minimized })}+\mathrm{E}_{\text {receptor(minimized })}\right.
$$

\subsection{Molecular dynamics}

To envisage the firmness and fitness of binding of the ligands inside the active site of 2B2A, we carried out MD simulations of three ligands, selected on their scoring functions, through Desmond module of Schrodinger 2015. The simulations were carried out under OPLS-2005 forcefield for 50ns. ${ }^{32}$ The solvation of the systems were done in orthorhombic boxes with explicit TIP4P water. Counter ions were added to neutralize the systems and simulations were performed under isothermal isobaric ensemble (NPT) ${ }^{33}$ having default temperature of $300 \mathrm{~K}$ and pressure of 1.013 bar. $^{34}$ RMSD plots for both the protein and the ligands reveals the relative stability of the lead inside its binding pocket of the target 2B2A. The Protein-Ligand (P-L) interaction histogram represents the interacting residues and their suitable interactions, which favours the binding.

\section{Results and discussion}

\subsection{Molecular Docking analysis against 2B2A receptor}

A series of catechin conformers and oligomers available from natural dietary sources were selected for the present study (Supporting Information, S2-S4). Monomers investigated include (+)-catechin (1), (-)-catechin (2), (+)epicatechin (3), (-)-epicatechin (4), (+)-gallocatechin (5), (-)-gallocatechin (6), (+)-epigallocatechin (7), (-)epigallocatechin (8), (+)-leucocyanidin (9) and (+)-leucopelargonidin (10). When subjected to virtual screening (2R,3S,4R)-2-[3,4-bis(oxidanyl)phenyl]-3,4-dihydro-2H-chromene-3,4,5,7-tetraol (9) depicted better docking with D-Score/G-Score of -7.2, with six hydrogen bond donors and seven hydrogen bond acceptors. Compounds 1-8 possess only two stereocenters at $2 \mathrm{C}$ and $3 \mathrm{C}$ whereas in compounds $\mathbf{9}$ and $\mathbf{1 0}$, there is an additional stereocentre at 4C with R-configuration of $2 \mathrm{H}$-chromene. This additional stereocentre generated by the introduction of an $-\mathrm{OH}$ group eventually increases the binding affinity (Supporting Information, S18). As evident from the interaction diagram, the newly introduced - $\mathrm{OH}(\mathrm{R})$ in $\mathbf{9}$ acts as hydrogen donor site, thereby coordinating with the negatively charged glutamic acid residue [Glu162] in the telomerase site. In addition, compound $\mathbf{9}$ exhibits a $\pi$-stacking interaction between its benzene ring and the positively charged arginine [Arg128]. The newly introduced -OH (S) group of $\mathbf{1 0}$ resulted in a slightly reduced specific hydrogen donating tendency since the interaction involved a 
polar glutamine [Gln168] . Interestingly compounds 7 and 5 with only two stereo centers showed better D-score/Gscore compared to 10. This observation suggests that the introduction of a hydroxy group enhances the ligand protein interaction while the stereochemistry of the chromene dictates specificity in binding (Supporting Information, S18).

It has recently been reported that artemisinin-derived dimers have better inhibiting capacity than the corresponding monomers. However, the exact mechanism of inhibition remains elusive till date. ${ }^{35}$ Since catechin oligomers are present in dietary sources, we next explored the potential of these compounds in telomerase inhibition. Of particular interest were the compounds having appreciable molecular weight range which may result in better docking due to the presence of multiple -OH groups. Interestingly, all except four $(\mathbf{1 3}, \mathbf{1 4}, 17$ and 20$)$ of the ten compounds 11-20 exhibited strong binding. Optimized geometries all the six ligands are depicted in Fig.1 and important interactions are noted in Table1. ProcyanidinA1(11) with twelve H-bond acceptors and $10 \mathrm{H}$-bond donors was found to be superior (G-score/D-score of -10.1.) to other compounds. This is in agreement with the recent reports on Cocoa procyanidins which showed selective cytotoxicity against epithelial ovarian cancer. ${ }^{36}$ It was observed that all the six dimers with five stereocentres showed better scores than the corresponding monomers, which may be attributed to the better suitability of the ligands in the pocket. It was also intriguing to find that the compounds $\mathbf{1 1}, \mathbf{1 9}$ and $\mathbf{1 2}$ with better scores share a common tendency of linking of two H-donor site -OH to the negatively charged glutamic acid [Glu162]. These results suggest that the effective binding demands the interaction of negatively charged glutamic acid [Glu162] with a H-donor of the ligand. Asn105 and Gln109 are the other two impotant aminoacids to which the high scored ligands show interaction by H-bond. Investigating the stereochemical aspects of the interaction diagrams (Fig.2), it is evident that the 2R, 3R configuration of chromene from epicatechin unit is significant for deciding both the effective binding with negatively charged glutamic acid as well as autofit of the ligand in the pocket.

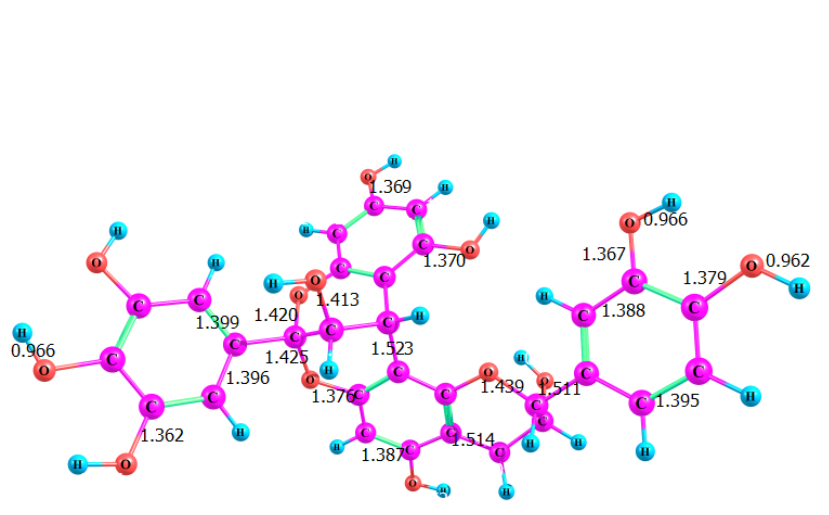

11 (Procyanidin A1)

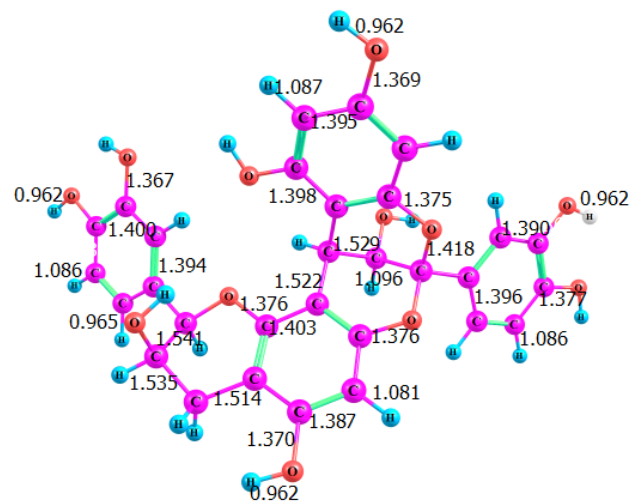

12 (Procyanidin A2) 

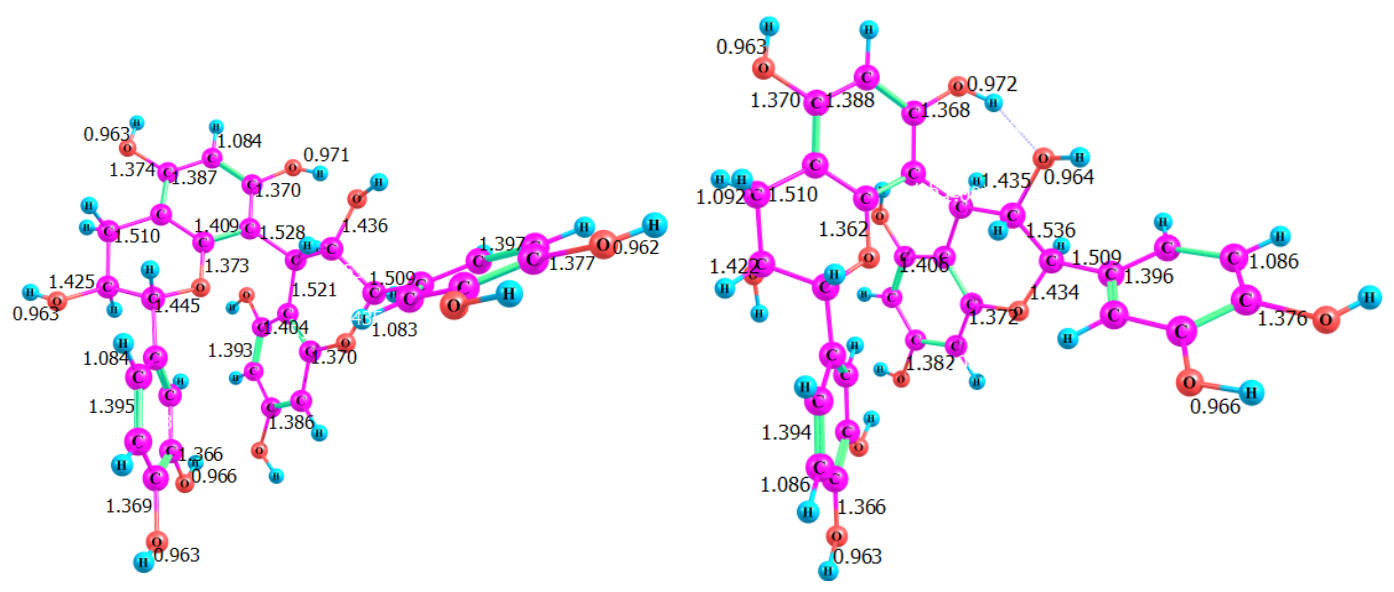

15 (Procyanidin B3)

16 (Procyanidin B4)

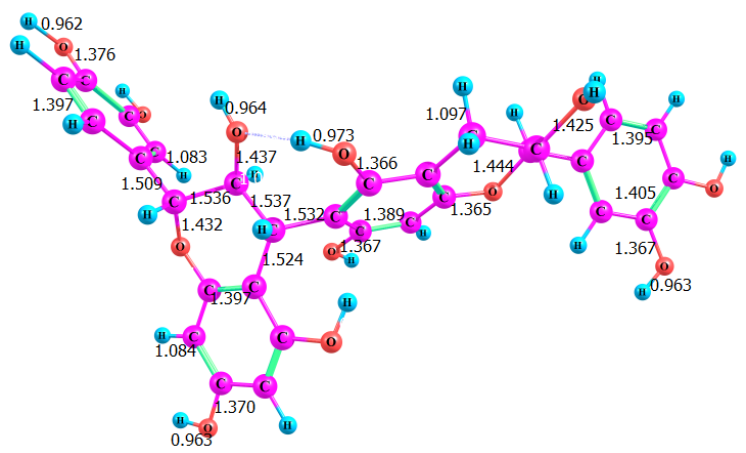

18 (ProcyanidinB6)

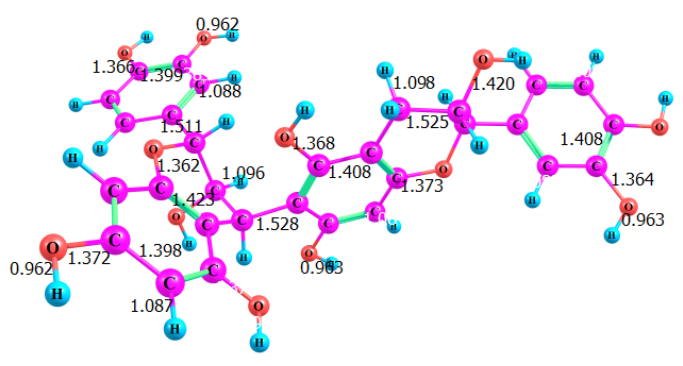

19 (Procyanidin B7)

Fig.1. Optimized geometries (B3LYP/6-311++G**) of high scored ligands

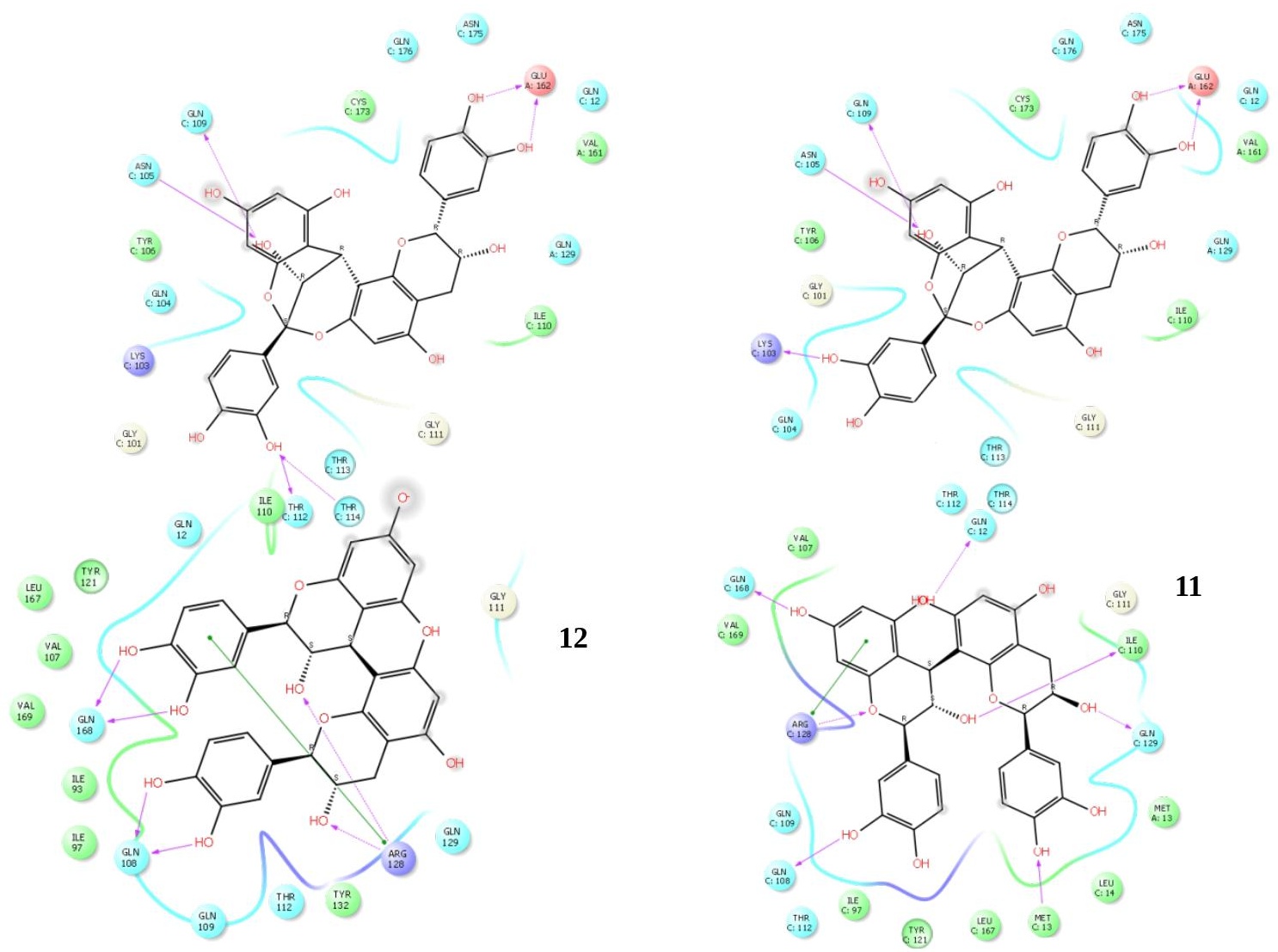




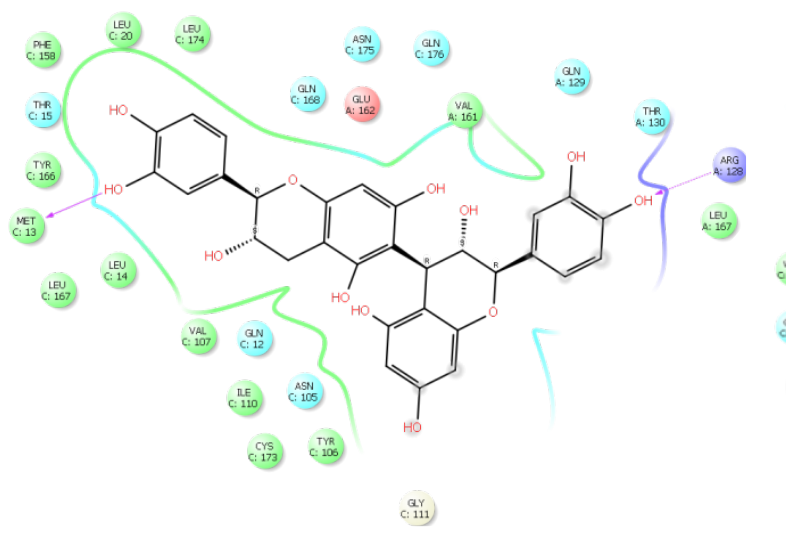

18

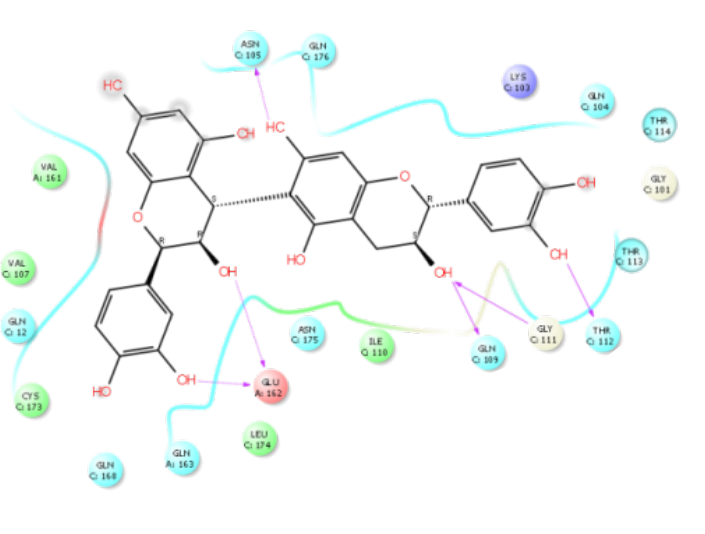

19

Fig.2. 11, 12, 15, 16, 18 and 19 in the binding site of 2B2A

Studies were further extended to analyse the properties of trimers using procyanidin C1 (21) and procyanidin C2 (22). Even though these compounds gave better score of -7.7 and -7.6 compared to monomers, the higher molecular weights make them less attractive drug candidates. Another aspect which renders these compounds unappealing is their inability to fit into the binding site due to their bulky size. In spite of the proximity of the two - $\mathrm{OH}$ in compound 22 to the glutamic acid residue, it was observed that the $2 \mathrm{R}$, $3 \mathrm{~S}$ configuration of the chromene hinders its effective binding, which confirms our finding that the stereochemistry of the ligand plays a critical role in its binding efficiency. The binding affinity were compared with the standard telomerase inhibitors: BIBR-1532 (a) and MST$312(\mathbf{b})^{37}$ and it was found that the D-score/G-score values are very low in a (-3.9/-3.9kcal/mol). b is sometimes better than a, but not as good as catechin dimers with a value of -6.6/-7.1 kcal/mol.

It has been reported elsewhere that parameters such as enthalpic and entropic components, hydrogen bond interactions, $\pi$-stacking interactions, etc. play a pivotal role in determining the binding efficiency between the ligand and receptor. ${ }^{38}$ In the present study we explored the contribution of 3D-positioning of intra ligand $\pi$-interaction between the monomers, in defining the binding of the dimers with the receptor. As the vertical alignment between 
the two aromatic systems gets perfected, the D-score also increases. It was observed that in the case of compound $\mathbf{1 1}$ with D-score and G-score of -10.1, the two $\pi$-systems are almost in perpendicularly eclipsed alignment to each other, resulting in an angle 0.0 between the centres of two systems. This increased the delocalisation leading to an increase in the electron density on terminal oxygens which was further supported by Natural Bond Orbital (NBO) analysis on terminal oxygens of the dimers (Fig.3, Table 1). NBO analysis revealed that the charge contribution from terminal oxygens is greatest in the case of 11, which increased the H-donating capacity. Eventually this adds to the superiority of $\mathbf{1 1}$ (procyanidin A1) as the hydrogen donor potential of the molecule has been enhanced. This property can be defined as "doppleganger effect". The word "doppelgänger" is often used in a more general sense to describe any person who physically or behaviorally resembles another person or shadow. In this context one of the aromatic systems stay behind the another as its shadow. Therefore it can be established that the stereochemical positioning of the two $\pi$-systems plays a major role in determining the binding affinity values.
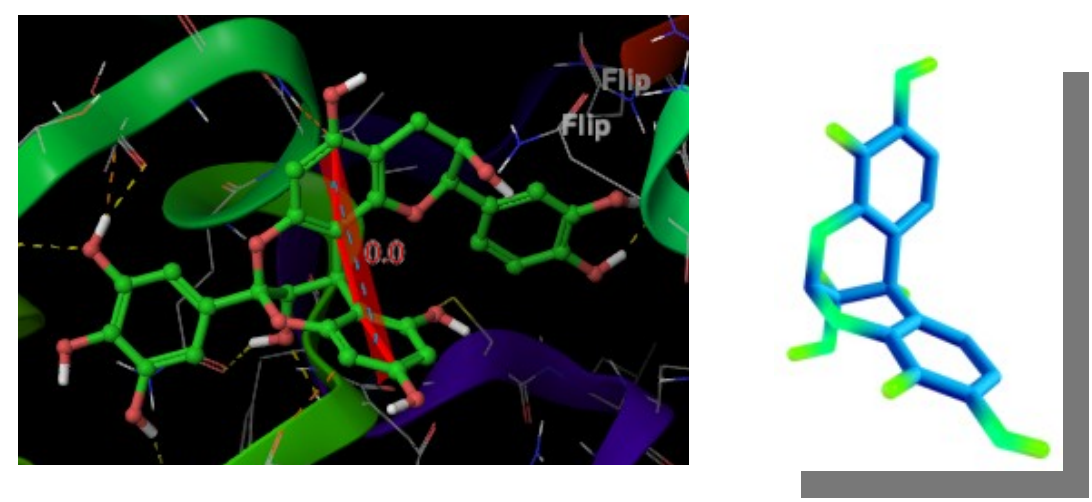

Fig.3. The alignment of two intra ligand $\pi$-systems in procyanidin A1

(11)

Table 1. The alignment angle and NBO analysis on terminal oxygens

\begin{tabular}{|l|c|c|c|c|c|c|c|c|c|c|}
\hline Entry & $\mathbf{1 1}$ & $\mathbf{1 2}$ & $\mathbf{1 3}$ & $\mathbf{1 4}$ & $\mathbf{1 5}$ & $\mathbf{1 6}$ & $\mathbf{1 7}$ & $\mathbf{1 8}$ & $\mathbf{1 9}$ & $\mathbf{2 0}$ \\
\hline $\begin{array}{l}\text { Alignment of } \\
\text { two } \text {-systems } \\
\text { (in terms of } \\
\text { angle) }\end{array}$ & 0.0 & 0.5 & -9.3 & -5.5 & 2.7 & -1.8 & 7.6 & -0.6 & -0.3 & 4.0 \\
\hline Dihedral angle & 94.9 & 99.9 & -152.1 & -164.2 & -44.8 & -54.2 & 128.7 & 82.6 & 125.3 & -158.5 \\
\hline $\begin{array}{l}\text { Natural charge } \\
\text { on terminal } \\
\text { oxygens }\end{array}$ & -0.72486 & -0.72399 & -0.75323 & -0.69940 & -0.72052 & -0.71178 & -0.69779 & -0.70334 & -0.75585 & -0.69871 \\
\cline { 2 - 11 } & -0.75258 & -0.75244 & -0.71757 & -0.69921 & -0.70564 & -0.69880 & -0.69819 & -0.70232 & -0.71964 & -0.69977 \\
\hline
\end{tabular}

\subsection{Analysis of ADME/T properties}

The ADME/T properties are also well suited for a drug as per pharmacokinetic parameters (Table 2). The molecular weights (M.W.) for the monomers and dimers are well within the range (130.0 to 725.0) with central nervous system toxicity (CNS) value of -2 . In the case of trimers the molecular weight is quite high with CNS of safe value (-2). All 
the monomers and dimers acts as drug like with \#stars of zero while that of trimers were found to be 2, which means the drug likeness is less for them. The hydrogen bond donors (HBD) are within 6 for monomers and dimers and the hydrogen bond acceptors (HBA) are less than 20 for all the three categories. The hydrophilic component of solvent accessible surface area (FISA) is well within the range (7.0 to 330.0) for the first two categories. The values for octanol/water partition cofficient ( $\mathrm{QPlogPo} / \mathrm{w}=-2.0$ to 6.5 ), aqueous solubility (QPlogS $=-6.5$ to 0.5 ) and binding to humam serum albumin (QPlogKhsa $=-1.5$ to 1.5 ) are within the range shows the binding and solubility features of all the ligands inside the blood circulation system. All the monomers and dimers are seem to be good candidates with Ro5 value of 0 or 1 and 100\% HOA(Human Oral Absorption). In the case of references a and $\mathbf{b}$, the MW, \#stars, CNS, FISA, HBA, HBD, QplogPo/w, QPlogS and QPlogKhsa are well within the range with minimum deviation from Ro5.

\subsection{MM-GBSA analysis and binding free energy prediction}

The free energy of binding (B.E.), $\Delta \mathrm{G}$ (bind), shows the interaction of the ligands within the binding pocket of 2B2A (Table2). As the value becomes more negative the complex is more stable. The B.E. is maximum $(-52.321 \mathrm{kcal} / \mathrm{mol})$ for procyanidin A1(11) with high score of $-10.2 \mathrm{kcal} / \mathrm{mol}$. The other ligands 19,12 and 15 also shows better B.E. compared to the standard MST-312 and all the ligands exhibit better B.E. compared to BIBR-1532. The calculattions shows that the B.E. is directly related to scoring function as expected.

Table 2. Pharmacokinetic parameters and G/D-Scores of 1-22

\begin{tabular}{|c|c|c|c|c|c|c|c|c|c|c|c|c|c|}
\hline No. & G-Score & D-Score & $\Delta \mathrm{G}_{\text {Bind }}$ & M.W. & \#stars & CNS & FISA & HBA & HBD & QPlogPo/w & QPlogS & QPlogKhsa & Ro5 \\
\hline 1 & -5.5 & -5.5 & -34.6 & 290.27 & 0 & -2 & 239.25 & 5.45 & 5 & 0.481 & -2.609 & -0.41 & 0 \\
\hline 2 & -4.4 & -4.4 & -22.7 & 290.27 & 0 & -2 & 239.25 & 5.45 & 5 & 0.481 & -2.609 & -0.41 & 0 \\
\hline 3 & -5.4 & -5.4 & -32.5 & 290.27 & 0 & -2 & 237.71 & 5.45 & 5 & 0.494 & -2.587 & -0.405 & 0 \\
\hline 4 & -5.0 & -5.0 & -30.7 & 290.27 & 0 & -2 & 237.71 & 5.45 & 5 & 0.494 & -2.587 & -0.405 & 0 \\
\hline 5 & -6.2 & -6.2 & -38.9 & 306.27 & 0 & -2 & 285.87 & 6.2 & 6 & -0.179 & -2.381 & -0.549 & 1 \\
\hline 6 & -5.5 & -5.5 & -34.8 & 306.27 & 0 & -2 & 285.87 & 6.2 & 6 & -0.179 & -2.381 & -0.549 & 1 \\
\hline 7 & -6.3 & -6.3 & -39.5 & 306.27 & 0 & -2 & 284.09 & 6.2 & 6 & -0.17 & -2.356 & -0.546 & 1 \\
\hline 8 & -3.0 & -3.0 & -17.5 & 306.27 & 0 & -2 & 284.09 & 6.2 & 6 & -0.17 & -2.356 & -0.546 & 1 \\
\hline 9 & -7.2 & -7.2 & -46.2 & 306.27 & 0 & -2 & 273.30 & 7.15 & 6 & -0.317 & -2.297 & -0.605 & 1 \\
\hline 10 & -5.9 & -5.9 & -36.7 & 290.27 & 0 & -2 & 223.86 & 6.4 & 5 & 0.322 & -2.466 & -0.488 & 0 \\
\hline 11 & -10.1 & -10.1 & -75.6 & 592.51 & 0 & -2 & 330.08 & 11.65 & 5 & -0.054 & -4.384 & -0.378 & 1 \\
\hline 12 & -8.2 & -8.2 & -73.9 & 576.51 & 0 & -2 & 382.95 & 10.9 & 5 & 0.638 & -4.665 & -0.208 & 1 \\
\hline 13 & -4.4 & -4.4 & -22.3 & 578.53 & 0 & -2 & 321.15 & 10.9 & 6 & 0.199 & -3.697 & -0.231 & 1 \\
\hline 14 & -4.8 & -4.7 & -25.6 & 578.53 & 0 & -2 & 321.19 & 10.9 & 5 & 0.244 & -3.76 & -0.218 & 1 \\
\hline 15 & -8.0 & -6.2 & -56.7 & 578.53 & 0 & -2 & 311.05 & 10.9 & 5 & 0.634 & -4.574 & -0.189 & 1 \\
\hline 16 & -8.2 & -8.1 & -65.1 & 578.53 & 0 & -2 & 309.58 & 10.9 & 5 & 0.619 & -4.587 & -0.201 & 1 \\
\hline 17 & -4.2 & -4.2 & -21.4 & 578.53 & 0 & -2 & 313.57 & 10.9 & 5 & 0.584 & -4.829 & -0.232 & 1 \\
\hline 18 & -7.5 & -7.5 & -48.5 & 578.53 & 0 & -2 & 304.41 & 10.9 & 5 & 0.668 & -4.872 & -0.229 & 1 \\
\hline 19 & -8.5 & -8.5 & -74.3 & 578.53 & 0 & -2 & 315.02 & 10.9 & 5 & 0.574 & -4.86 & -0.236 & 1 \\
\hline 20 & -4.9 & -4.9 & -26.5 & 578.53 & 0 & -2 & 302.54 & 10.9 & 5 & 0.681 & -4.848 & -0.225 & 1 \\
\hline 21 & -7.6 & -7.2 & -49.6 & 866.78 & 2 & -2 & 566.90 & 16.35 & 15 & -0.063 & -4.236 & -0.241 & 2 \\
\hline 22 & -7.7 & -7.0 & -47.4 & 866.78 & 2 & -2 & 570.98 & 16.35 & 15 & 0.571 & -6.093 & -0.201 & 3 \\
\hline
\end{tabular}

+2; FISA(Hydrophilic component of total solvent accessable area): 7.0-333.0; HBA(Hydrogen bond acceptor): 2.0-20.0; HBD(hydrogen bond donor): 0.0-6.0; QPlogPo/w(octanol/water partition coefficient): -2.0-6.5; QPlogS(Aqueous solubility): -6.5-0.5; QPlogKhsa(binding to human serum albumin): -1.5-1.5; Ro5(Number of violations of Lipinski's rule of five): maximum is 4.

\subsection{MD simulation analysis}


The flexibility in conformation and stability of the ligands inside the active site of the receptor (with 11, 12 and 19) were established by molecular dynamics (MD) analysis under OPLS2005 force field at 50 nanosecond (ns). To resolve the stability and conformational flexibilty of the protein-ligand interactions, Root Mean Square Deviation (RMSD), Root Mean Square Fluctuation (RMSF), Protein-Ligand (P-L) contacts, ligand torsion profile of the protein-ligand complex of highest scores were determined. Fig.4 \& 5 shows the average RMSD of the C $\alpha$ atoms of the protein 2B2A(left Y-axis) and the ligand (right Y-axis). RMSD of the ligand heavy atoms is measured by aligning the protein-ligand complex on the receptor backbone of the reference. The magnitude of fluctuations indicates that the complexes attained stable conformations after equilibration and how stable the ligands are with refer to the

active

in 11,12

values

lower than

the

denotes

are

to the

site even

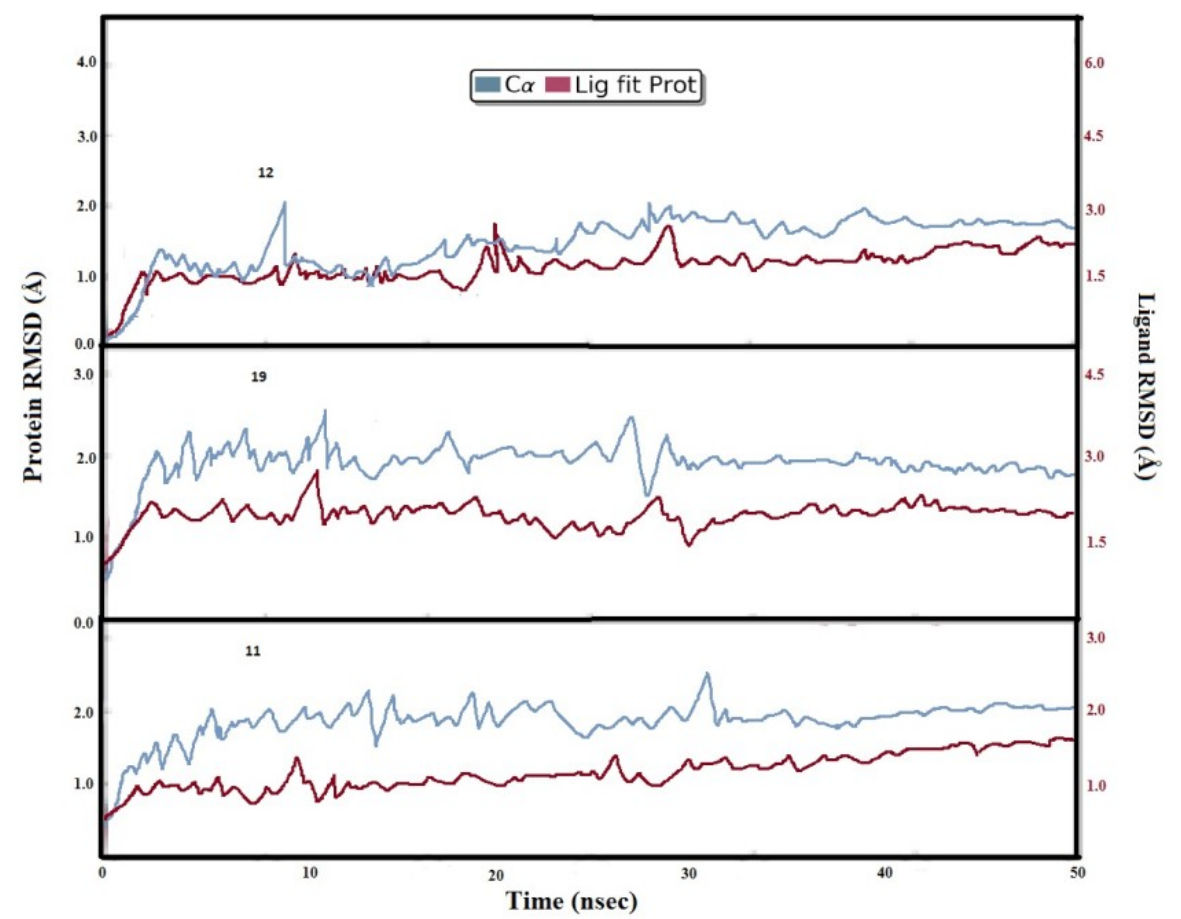

protein and its binding pocket and 19. The predicted are significantly the RMSD of protein, which the ligands strongly bind initial binding after 50ns.

Fig.4. RMSD plots of C $\alpha$ of 2B2A and ligands 11, 19 and 12 

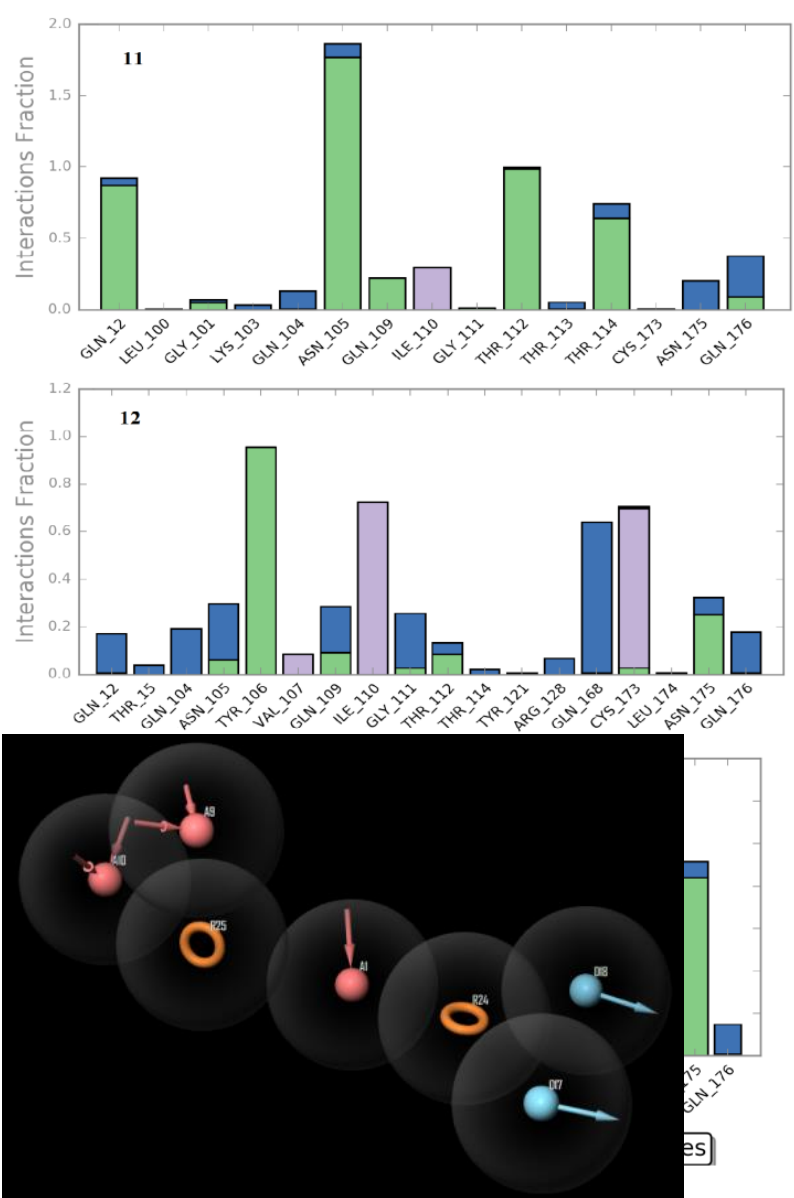

Fig.5. P-L interactions of 11, 12 and 19

P-L interactions throughout the simulations can be categorized and summarised in Fig.5. The stacked bar charts for hydrogen bonds, hydrophobic, ionic and water bridges are normalized over the course of the trajectory. H-bond play a significant role in ligand binding because of their strong influence on drug specificity, metabolization and absorption. H-bonds predominates and most of them last for more than $80 \%$ time of simulation. In 11, there are six H-bond donors and two H-bond acceptors. Among these H-bond with E162 lasts for 100\%, 98\% and 86\%; N105 lasts for $98 \%$ and T112 lasts for $98 \%$ of simulation time. While in 19, there are $5 \mathrm{H}$-donors and only two $\mathrm{H}$ acceptors, among these H-bond with Q109 (88\%), N105 (90\%), Q12(80\%), Q168 (92\%), N175 (84\%), Q163 (96\%) last for the starting to end of the simulation time. But in 12, there are only two H-donors to E162 last for $96 \%$ and Y106 for 95\%. The contacts confirmed the interaction with glutamate moiety, E162 and Q163 upto 100\% of simulation time and gives the stability to P-L complex. In order to generate new chemotypes that are able to make similar interactions, we have done 3D pharmacophore model for dimers (11-20) using seven-point hypothesis based on best allignment and common features (Fig.6). The hypothesis includes two donors, three acceptors and two rings in common to fit all the 10 ligands (11-20). This data is helpful design new inhibitors for telomerase more accurately. 
Fig.6. 3D pharmacophore model generated for the ligands 11-20

\section{Conclusion}

In summary, we investigated the stereochemical aspects of telomerase inhibition by Catechin analogues and it's oligomers. It was found that the catechin ligands bind effectively to the negatively charged glutamic acid residue present in the binding site of the TEN domain of telomerase. Among the various compounds studied, those with $\mathrm{H}-$ donors which interacted strongly with this glutamic acid residue were found to exhibit strong binding. This observation ascertains the fact that the ligand binding to this glutamic acid ensures better interaction with the protein. The catechin dimers having $2 \mathrm{R}, 3 \mathrm{R}$ configuration in the chromene ring were found to have strong interaction with telomearse, which was exemplified by the superiority of the dimer procyanidin A1 (11) in the binding with the protein. Molecular dynamics simulations prove the stability of protein-ligand complex. RMSD, RMSF and P-L contacts supports the stability of P-L complex. We envisage that these stereochemical and mechanistic revelations regarding the binding interactions of catechin ligands and the corresponding dimers with telomearse could open up new avenues in chemotherapy.

\section{Conflict of interst}

There is no conflict of interest

\section{Acknowledgements}

The authors thank IIITMK, Trivandrum for providing research facilities.

\section{References}

1. Blanco R, Munoz P, Klatt P, Flores JM, Blasco MA (2007) Telomerase abrogation dramatically accelerates TRF2-induced epithelial carcinogenesis. Genes Dev 21:206-220.

2. Jerry WS, Ying Z, Eiso H, Woodring EW (2001) Telomerase and cancer. Hum Mol Genet 10:677-685.

3. Mohammad AJ, Shakeel AA, Mohammed HA, Jerry WS (2016) Roles of telomeres and telomerase in cancer, and advances in telomerase targeted Therapies. Genome Medicine 8:1-18.

4. Zhang Q, Kim NK, Feigon J (2001) Architecture of human telomerase RNA. PNAS 108:20325-20332.

5. Huang J, Brown AF, Wu J, Bley CJ, Rand DP, Wu L, Zhang R, Chen J, Lei M (2014) Structural basis for protein-RNA recognition in telomerase. Nat Struct and Mol Biol 21:507-512.

6. Jacobs SA, Podell ER, Cech TR (2006) Crystal structure of the essential N-terminal domain of telomerase reversetransciptase. Nat Struct and Mol Biol 13:218-225.

7. Mitchell M, Gillis A, Futahashi M, Fujiwara H, Skordalakes E (2010) Structural basis for telomerase catalytic subunit TERT binding to RNA template and telomeric DNA. Nat Struct and Mol Biol 17:513519 . 
8. Hapangama DK., Turner MA, Drury JA, Quenby S, Saretzki G, Martin-Ruiz C, Von Zglinicki T (2008) Endometriosis is associated with aberrant endometrial expression of telomerase and increased telomere length. Hum Reprod 23: 1511-1519.

9. Hapangama DK, Kamal A, Saretzki G (2016) Implications of telomeres and telomerase in endometrial pathology. Hum Reprod Update 23:166-187.

10. Valentijn AJ, Saretzki G, Tempest N, Critchley HOD, Hapangama DK (2015) Human endometrial epithelial telomerase is important for epithelial proliferation and glandular formation with potential implications in endometriosis. Hum Reprod 30:2816-2828.

11. Valentijn AJ, Palial K, Al-lamee H, Tempest N, Drury J, Von Zglinicki T, Saretzki G, Murray P, Gargett CE, Hapangama DK (2013) SSEA-1 isolates human endometrial basal glandular epithelial cells: phenotypic and functional characterization and implications in the pathogenesis of endometriosis. Hum Reprod 28:2695-2708.

12. Wang J, Zhao C, Zhao A, Li M, Ren J, Qu X (2015) New insights in amyloid beta interactions with human telomerase. J Am Chem Soc 137:1213-1219.

13. Steczkiewicz K, Zimmermann MT, Kurcinski M, Lewis A, Dobbs D, Kloczkowski A, Jernigan RL, Kolinski A, Ginlski K (2011) Human telomerase model shows the role of the TEN domain in advancing the double helix for the next polymerization step. PNAS 108:9443-9448.

14. Maji B, Bhattacharya S (2014) Advances in the molecular design of potential anticancer agents via targeting of human telomeric DNA. Chem Commun 50: 6422-6438. doi:10.1039/C4CC00611A

15. Sandin S, Rhodes D (2014) Telomerase structure. Curr Opin Struct Biol 25:104-110.

16. White LK, Wright WE, Shay J W (2001) Telomerase inhibitors. Trends Biotechnol 19:114-120.

17. Saretzki G (2003) Telomerase inhibition as cancer therapy. Cancer Lett 194, 209-219.

18. Corey DR (2002) Telomerase inhibition, oligonucleotides and clinical trials. Oncogene 21:631-637.

19. Cunningham AP, Love WK, Zhang RW, Andrews LG, Tollefsbol TO (2006) Telomerase inhibition in cancer therapeutics: Molecular-based approaches. Curr Med Chem 13:2875-2888.

20. Andrews LG, Tollefsbol TO (2008) Methods of telomerase inhibition. Mol Biol 405:1-8.

21. Talari FS, Bagherzadeh K, Golestanian S, Jarstfer M, Amanlou M (2015) Potent Human Telomerase Inhibitors: Molecular Dynamic Simulations, Multiple Pharmacophore-Based Virtual Screening, and Biochemical Assays. J Chem Inf Model 55:2596-2610.

22. Chen JL, Sperry J, Ip NY, Brimble MA (2011) Natural products targeting telomerase maintenance. Med Chem Commun 2:229-245.

23. Cohn EP, Wu KL, Pettus TRR, Reich NO (2012) A new strategy for detection and development of tractable telomerase inhibitors. J Med Chem 55:3678-3686. 
24. Shoichet BR (2004) Virtual screening of chemical libraries. Nature 432:862-865.

25. Chahar MK, Sharma N, Dobhal MP, Joshi YC (2011) Flavonoids:A versatile source of anticancer drugs. Pharmacogn Rev 5:1-12.

26. Menichincheri M, Ballinari D, Bargiotti A, Bonomimi L, Ceccarelli W, D’Alessio R, Fretta A, Moll J, Polucci P, Soncini C, Tibolla M, Trosset J, Vanotti E (2004) Catecholic flavonoids acting as telomerase inhibitors. J Med Chem 47:6466-6475.

27. Gadkar PV, Balaraman M (2015) Catechins:Sources, extraction and encapsulation: A review, Food Bioprod Process 93:122-138.

28. Imad N, Oh-hashi F, Oh-hara T, Feng WY, Johnston J, Chan K, Tsuruo T (2003) Blocking telomerase by dietary polyphenols is a major mechanism for limiting the growth of human cancer cells in vitro and in vivo. Cancer Res 63:824-830.

29. Takanashi K, Manato S, Kiriko M, Chisato I, Kazuya T, Koichiro K, Shogo S, Narumi K. Mikihiro I, Miyuki K, Yasunao H, Sei-ichi K, Koji U, Hiroshi F, Hidefumi M (2017) Epicatechin oligomers longer than trimers have anti-cancer activities, but not the catechin counterparts. Sci Reports 7:77917802.

30. Sastry GM, Adzhigirey M, Day T, Annabhimoju R, Sherman W (2013) Protein and ligand preparation: Parameters, protocols, and influence on virtual screening enrichments. J Comput Aid Mol Des 27:221-234.

31. Halgren TA, Murphy RB, Friesner RA, Beard HS, Frye Ll, Pollard WT, Banks JL (2004) Glide: A new approach for rapid, accurate docking and scoring. J Med Chem 47:1750-1759.

32. Guo Z, Mohanty U, Noehre J, Sawyer TK, Sherman W, Krilov G (2010) Probing the $\alpha$-Helical Structural Stability of Stapled p53 Peptides: Molecular Dynamics Simulations and Analysis. Chem Biol Drug Des 75:348-359.

33. Azam MA, Jupudi S (2017) Extra precision docking, free energy calculation and molecular dynamics studies on glutamic acid derivatives as MurD inhibitors. Comput Biol Chem 69:55-63.

34. Singh SP, Gupta D (2017) Discovery of potential inhibitor against human acetylcholinesterase: a molecular docking and molecular dynamics investigation. Comput Biol Chem 68:224-230. 
35. He R, Mott BT, Rosenthal AS, Genna DT, Posner GH, Arav-Boger R (2011) An Artemisinin-Derived Dimer Has Highly Potent Anti-Cytomegalovirus (CMV) and Anti-Cancer Activities. PLOS ONE 6: e24334.

36. Shruti T, Aparna K (2016) Effect of Procyanidin-rich Extract from Natural Cocoa Powder on Cellular Viability, Cell Cycle Progression, and Chemoresistance in Human Epithelial Ovarian Carcinoma Cell Lines. Pharmacogn Mag 12(Suppl 2): S109-S115.

37. Waghorn PA, Jackson MR, Gouvernuer V, Vallis KA (2017) Targeting telomerase with radiolabeled inhibitors. Eur J Med Chem 125:117-129. doi: 10.1016/j.ejmech.2016.09.028

38. Bissantz C, Kuhn B, Stahl MA (2010) Medicinal Chemist’s Guide to Molecular Interactions. J Med Chem 53:5061-5084. 
